\title{
Underground measurement at LUNA found no evidence for a low-energy resonance in the ${ }^{6} \mathrm{Li}(\mathrm{p}, \gamma)^{7} \mathrm{Be}$ reaction
}

\author{
Denise Piatti ${ }^{1, *}$ for the LUNA Collaboration \\ ${ }^{1}$ Univerisità degli Studi di Padova and INFN Division of Padova, Via Marzolo 835136 Padova (Italy)
}

\begin{abstract}
The ${ }^{6} \mathrm{Li}(\mathrm{p}, \gamma)^{7} \mathrm{Be}$ reaction is involved in all three main nucleosynthesis scenarios: Big Bang Nucleosynthesis, the interaction of cosmic rays with interstellar matter, and stellar nucleosynthesis.

Conflicting experimental results have been reported in literature for the ${ }^{6} \mathrm{Li}(\mathrm{p}, \gamma){ }^{7} \mathrm{Be}$ reaction cross section trend at astrophysical energies. A recent direct measurement found a resonance-like structure at $E_{\mathrm{c} . \mathrm{m} .}=195 \mathrm{keV}$, corresponding to an excited state at $E_{\mathrm{x}} \sim 5800 \mathrm{keV}$ in ${ }^{7} \mathrm{Be}$ which, however, has not been confirmed by either theoretical calculations or other direct measurements. In order to clarify the existence of this resonance, a new experiment was performed at the Laboratory for Underground Nuclear Astrophysics, located deep underground at Laboratori Nazionali del Gran Sasso (Italy). The ${ }^{6} \mathrm{Li}(\mathrm{p}, \gamma)^{7} \mathrm{Be}$ cross section was measured in the energy range $E_{\text {c.m. }}=60-350 \mathrm{keV}$ with unprecedented sensitivity and no evidence for the alleged resonance was found.
\end{abstract}

\section{Introduction}

According to simulations of the Galaxy chemical evolution most of the solar system lithium was provided by low-mass stars [1] while less than half of it was produced by Big Bang Nucleosynthesis (BBN) [2,3] or Galactic cosmic rays interacting with interstellar matter.

The predicted $\mathrm{BBN}^{6} \mathrm{Li} /{ }^{7} \mathrm{Li}$ isotopic ratio is $\sim 10^{-5}$ [4], significantly lower than the solar system value of 0.08 [5]. Very low ${ }^{6} \mathrm{Li} /{ }^{7} \mathrm{Li}$ values are expected for neutrino nucleosynthesis [6] and for stellar sources as well. In contrast, in case of Galactic or structure formation cosmic rays the ${ }^{6} \mathrm{Li} /{ }^{7} \mathrm{Li}$ production ratio is close to unity [7].

The ${ }^{6} \mathrm{Li} /{ }^{7} \mathrm{Li}$ isotopic ratio has been indeed proposed as a tool to constrain non-standard lithium production mechanisms [8] and pollution of stellar atmospheres [9] in the context of the cosmological lithium problem,

The ${ }^{6} \mathrm{Li}(\mathrm{p}, \gamma)^{7} \mathrm{Be}$ reaction plays a key role in determining the stellar ${ }^{6} \mathrm{Li} /{ }^{7} \mathrm{Li}$. The ${ }^{6} \mathrm{Li}(\mathrm{p}, \gamma){ }^{7} \mathrm{Be}$ reaction may indeed not only deplete ${ }^{6} \mathrm{Li}$ but also convert some of it to ${ }^{7} \mathrm{Li}$, through ${ }^{7}$ Be radioactive decay.

Measurements of the ${ }^{6} \mathrm{Li}(\mathrm{p}, \gamma)^{7} \mathrm{Be}$ reaction cross section at low energies have reported inconsistent results on the slope of the astrophysical $S$-factor $[10,11]$. Moreover, the positive slope reported by [12] was interpreted as a new resonance at $E_{\mathrm{c} . \mathrm{m} .}=195 \mathrm{keV}$, corresponding to an excited level at $E_{x} \approx 5800 \mathrm{keV}$ with $J^{\pi}=\left(1 / 2^{+}, 3 / 2^{+}\right)$and $\Gamma_{p} \approx 50 \mathrm{keV}$. No evidence for such a resonance was found in the ${ }^{3} \mathrm{He}\left({ }^{4} \mathrm{He}, \gamma\right){ }^{7} \mathrm{Be}$ reaction at $E_{\mathrm{c} . \mathrm{m} .}=4210 \mathrm{keV}$ as reported in recent comprehensive study [13].

\footnotetext{
*e-mail: denise.piatti@pd.infn.it
} 
None of the theoretical calculations of the ${ }^{6} \mathrm{Li}(\mathrm{p}, \gamma){ }^{7} \mathrm{Be} S$-factor are able to reproduce the newly-reported resonance [14, 15, and references therein], unless this is added ad-hoc to reproduce the experimental data [16].

\section{Experimental Setup}

We performed a new experiment [17] at the Laboratory for Underground Nuclear Astrophysics (LUNA), located deep underground at Laboratori Nazionali del Gran Sasso (Italy) [18].

A schematic view of the experimental setup is shown in Fig.1. The LUNA-400 accelerator

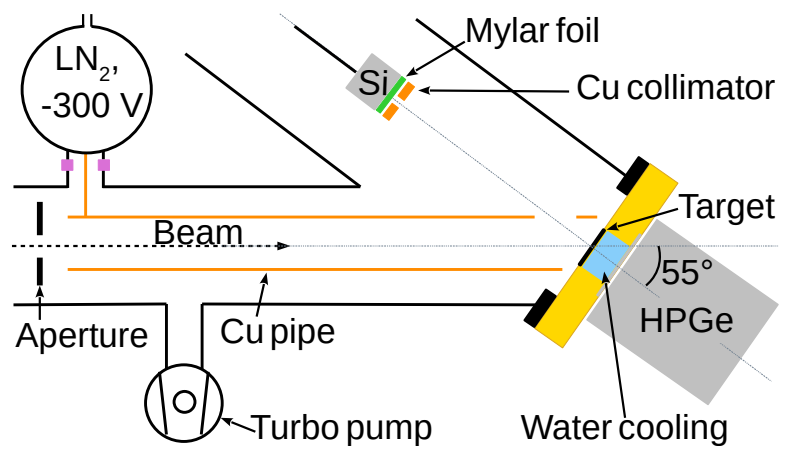

Figure 1: Sketch of the experimental setup used for the measurement of the ${ }^{6} \operatorname{Li}(\mathrm{p}, \gamma)^{7} \mathrm{Be}$ cross section at LUNA [17].

[19] high-intensity proton beam was collimated by a $3 \mathrm{~mm}$ diameter aperture and delivered through a copper pipe to the target, mounted at $55^{\circ}$ with respect to the beam direction. The $\mathrm{Cu}$ tube was used both as a cold trap and for secondary electron suppression. Three evaporated targets (thicknesses $100-200 \mu \mathrm{g} / \mathrm{cm}^{2}$ ) were made from ${ }^{6} \mathrm{Li}_{2} \mathrm{WO}_{4}$ powder and one (thickness $20 \mu \mathrm{g} / \mathrm{cm}^{2}$ ) was made using ${ }^{6} \mathrm{Li}_{2} \mathrm{O}$ powder. The ${ }^{6} \mathrm{Li}$ isotopic enrichment level was $95 \%$ for all targets, which were water cooled during irradiation in order to limit target degradation [17].

A High-Purity Germanium (HPGe) detector positioned in close geometry to the target and at $55^{\circ}$ with respect to the beam direction was used to detect ${ }^{6} \mathrm{Li}(\mathrm{p}, \gamma)^{7} \mathrm{Be}$ reaction $\gamma$ rays. To detect the $\alpha$ and ${ }^{3} \mathrm{He}$ particles from the ${ }^{6} \mathrm{Li}(\mathrm{p}, \alpha)^{3} \mathrm{He}$ reaction concurrently with the gamma rays from the ${ }^{6} \mathrm{Li}(\mathrm{p}, \gamma)^{7} \mathrm{Be}$ reaction, a silicon detector was installed at $125^{\circ}$ from the beam direction. Efficiencies for both detectors were obtained using GEANT simulations, fine tuned through the comparison with experimental results [17].

\section{Analysis and Results}

To make consistency checks and verify results are unaffected by systematic effects, a measurement of the ${ }^{6} \mathrm{Li}(\mathrm{p}, \gamma)^{7} \mathrm{Be}$ and ${ }^{6} \mathrm{Li}(\mathrm{p}, \alpha){ }^{3} \mathrm{He}$ excitation functions was performed for each target in the whole dynamic range of the LUNA-400 accelerator [17].

The ${ }^{6} \mathrm{Li}(\mathrm{p}, \gamma)^{7} \mathrm{Be}$ experimental yield was calculated from the sum of the contributions from the direct capture to the ground state $\left(\gamma_{0}\right)$ and to the $429 \mathrm{keV}$ excited state of ${ }^{7} \mathrm{Be}\left(\gamma_{1}\right)$.

For the calculation of the ${ }^{6} \mathrm{Li}(\mathrm{p}, \gamma)^{7} \mathrm{Be}$ reaction $S$-factor, we adopted a relative approach [17]: the (p, $\gamma$ ) yield was normalized at each energy to the (p, $\alpha$ ) yield. This ratio can be expressed in terms of the (p, $\gamma)$ and $(\mathrm{p}, \alpha) S$-factors. We adopted for the ${ }^{6} \mathrm{Li}(\mathrm{p}, \alpha)^{3} \mathrm{He}$ reaction 
the $S$-factor parametrization from [20]. For the (p, $\alpha)$ channel, the angular distribution coefficients $A_{k}$ and related uncertainties were taken from [21, and references therein]. For the $(\mathrm{p}, \gamma)$ channel we adopted the theoretical angular distribution described in [14]. Finally the measured $S$-factor was corrected for electron screening using the adiabatic approximation [22] with screening potential $\mathrm{U}_{e}=273 \mathrm{eV}$ [20].

Our $S$-factor data have a monotonic dependence on the energy and show no evidence of the resonance reported by [12], see Fig.2. The ${ }^{6} \mathrm{Li}(p, \gamma)^{7} \mathrm{Be}$ reaction cross section was measured in the energy range $60-350 \mathrm{keV}$ with $\leq 2 \%$ statistical and $12 \%$ systematic uncertainty. An R-matrix fit of our data and the data from [23] was performed and used to calculate a new ${ }^{6} \mathrm{Li}(p, \gamma)^{7} \mathrm{Be}$ reaction rate. The proposed reaction rate is $9 \%$ lower than NACRE [24] and $33 \%$ higher than reported in NACREII [16] at $2 \mathrm{MK}$, relevant for ${ }^{6} \mathrm{Li}$ depletion in premain sequence stars, and the reaction rate uncertainty has been significantly reduced [17], see Fig.3.

The result of a recent indirect study supports LUNA extrapolation for the ${ }^{6} \mathrm{Li}(p, \gamma){ }^{7} \mathrm{Be}$ $S$-factor [25].

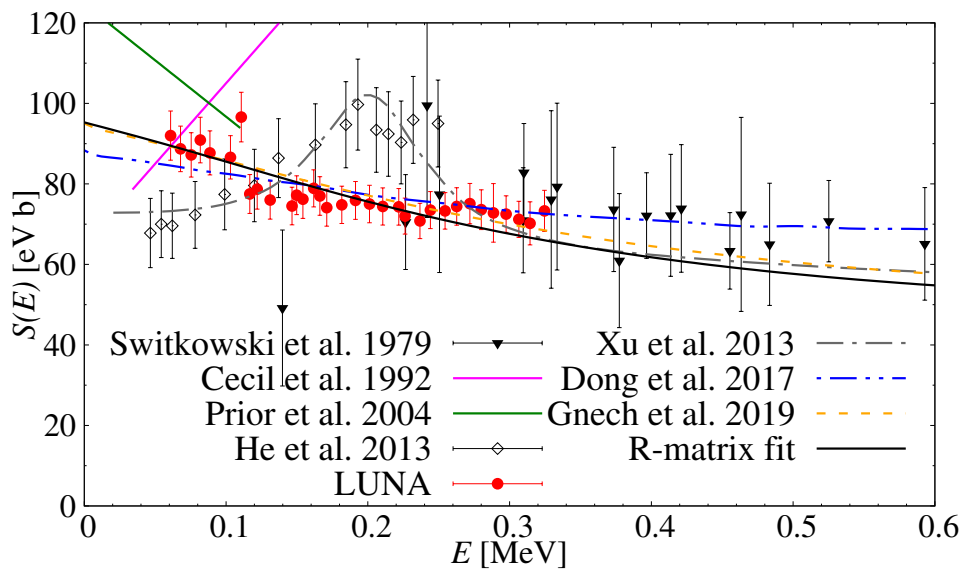

Figure 2: Astrophysical S-factor for the ${ }^{6} \mathrm{Li}(p, \gamma)^{7} \mathrm{Be}$ reaction as obtained by LUNA in red [17]. Previous experimental data and theoretical evaluations are also shown for comparison. The solid black line represents an R-matrix fit of LUNA data and data from [23]. 


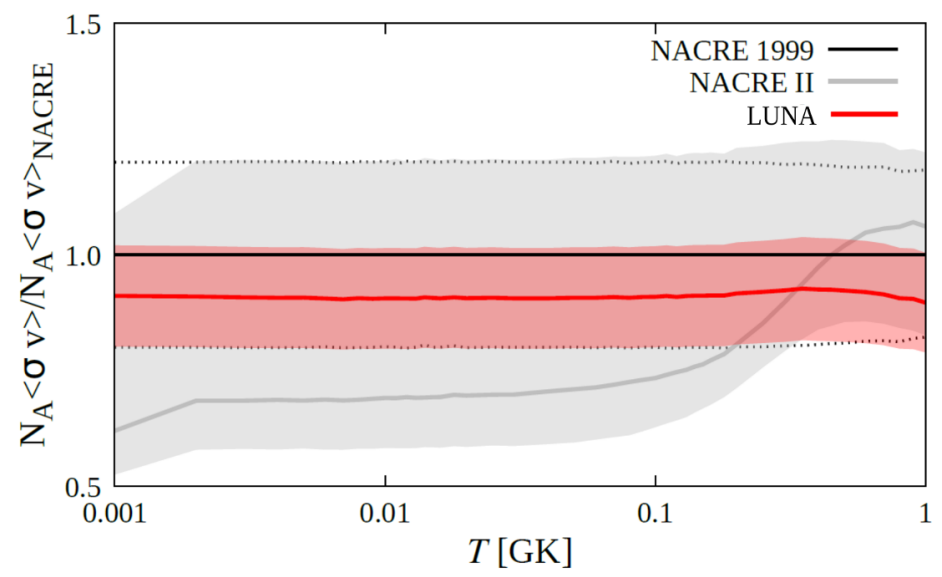

Figure 3: Reaction rate for the ${ }^{6} \mathrm{Li}(p, \gamma)^{7} \mathrm{Be}$ reaction, normalized to the NACRE rate [24]. The NACRE II rate [16] is also shown for comparison. Dashed lines represent the uncertainty on the NACRE rate, while shaded areas represent the uncertainties from LUNA experiment (red) and from NACRE II (grey).

\section{References}

[1] N. Prantzos et al., Astronomy and Astrophysics 542, A67 (2012)

[2] C. Pitrou et al., Physics Reports 754, 1 (2018)

[3] B. D. Fields et al.,Journal of Cosmology and Astroparticle Physics 2020, 010 (2020)

[4] M. Anders et al., Physical Review Letters 113, 042501 (2014)

[5] K. Lodders, Astrophysical Journal 591, 1220 (2003)

[6] A. Sieverding et al., Astrophysical Journal 876, 151 (2019)

[7] B. D. Fields and T. Prodanović, Astrophysical Journal 623, 877 (2005)

[8] J. C. Howk et al., Nature 489, 121 (2012)

[9] G. Harutyunyan et al., Astronomy and Astrophysics 618, A16 (2018)

[10] F. Cecil et al., Nuclear Physics A 539, 75 (1992)

[11] R. M. Prior et al., Physical Review C 70, 055801 (2004)

[12] J. He et al., Physics Letters B 725, 287 (2013)

[13] T. Szücs et al., Physical Review C 99, 055804 (2019)

[14] A. Gnech and L. E. Marcucci, Nuclear Physics A 987, 1 (2019)

[15] G. X. Dong et al., Journal Physics G Nuclear and Particle Physics 44, 045201 (2017)

[16] J. Huang et al., Atomic Data and Nuclear Data Table 96, 824 (2010)

[17] D. Piatti et al., Physical Review C 102, (2020)

[18] F. Cavanna and P. Prati, International Journal of Modern Physics A 33, 1843010 (2018)

[19] A. Formicola et al., Nuclear Instruments and Methods in Physics Research 507, 609 (2003)

[20] J. Cruz et al., Physics Letters B 624, 181 (2005)

[21] C. Brune et al., Nuclear Instruments and Methods in Physics Research A 389, 421 (1997)

[22] H. J. Assenbaum, K. Langanke and C. Rolfs, Zeitschrift für Physik A Atomic Nuclei 327, 461 (1987) 
[23] Z. Switkowski et al., Nuclear Physics A 331, 50 (1979)

[24] C. Angulo et al., Nuclear Physics A 656, 3 (1999)

[25] G.G. Kiss et al., Physical Review C 104, 015807 (2021) 